\title{
Transcriptional Levels of Autophagy and UPR Markers in the Brain and Liver of Pregnant Rats
}

\author{
Saeed Alizadeh ${ }^{1}$, Ghasem Ghasempour ${ }^{1,2}$, Elnaz Golestaneh ${ }^{3}$, Yasaman Safian Isfahani ${ }^{3}$, Arya Emami \\ ${ }^{4}$, Tania Dehesh ${ }^{5}$, Sayed Mohammad Shafiee ${ }^{6}$, Ali Akbar Owji ${ }^{1}$ and Shahla Shojaei $\mathbb{E}^{3,7,{ }^{*}}$ \\ ${ }^{1}$ Department of Clinical Biochemistry, Research Center for Psychiatry and Behavioral Sciences, Shiraz University of Medical Sciences, Shiraz, Iran \\ ${ }^{2}$ Department of Clinical Biochemistry, School of Medicine, Iran University of Medical Sciences, Tehran, Iran \\ ${ }^{3}$ Department of Clinical Biochemistry, School of Pharmacy and Pharmaceutical Sciences, Isfahan University of Medical Sciences, Isfahan, Iran \\ ${ }^{4}$ Department of Psychology, Faculty of Health Sciences, York University, Toronto, Canada \\ ${ }^{5}$ Department of Biostatistics and Epidemiology, School of Public Health, Kerman University of Medical Sciences, Kerman, Iran \\ ${ }^{6}$ Department of Biochemistry, School of Medicine, Shiraz University of Medical Sciences, Shiraz, Iran \\ ${ }^{7}$ College of Pharmacy, Rady Faculty of Health Sciences, University of Manitoba, Winnipeg, Canada \\ "Corresponding author: College of Pharmacy, Rady Faculty of Health Sciences, University of Manitoba, Winnipeg, Canada. Email: shojaeis2019@gmail.com
}

Received 2020 December 01; Revised 2020 December 29; Accepted 2021 January 05.

\begin{abstract}
Background: Pregnancy is associated with oxidative stress that results in endoplasmic reticulum (ER) stress and unfolded protein response (UPR). Prolonged-unalleviated ER stress causes the activation of the autophagy pathway via UPR. Expression of genes encoding glucose-regulated protein 78 (GRP78) and BECLIN1 are induced in UPR and autophagy.

Objectives: We studied the mRNA expression of the aforementioned genes in the liver and brain of Nulligravida versus saline and ethanol-treated pregnant rats.

Methods: Control pregnant rats were orally treated with normal saline, and test animals received ethanol $250 \mathrm{mg} / \mathrm{kg}$ or resveratrol $120 \mathrm{mg} / \mathrm{kg}$ from day 1 to day 21 of gestation. Nulligravida rats treated by saline comprised the non-pregnant control group. On day 21, mRNAs encoding GRP78 and BECLIN1 were extracted from the liver and brain tissues and assessed using real-time PCR.

Results: Our results showed that the level of transcripts encoding GRP78 and BECLIN1 was higher in the liver of pregnant rats compared to Nulligravida ones. Further, ethanol decreased the mRNA levels of GRP78 and BECLIN1 in the liver of pregnant rats, an effect that was reversed by resveratrol. Levels of GRP78 transcripts were decreased, and those of BECLIN1 remained unchanged in the brain of ethanol exposed pregnant rats.

Conclusions: Levels of mRNAs for GRP78 and BECLIN1 are up-regulated during pregnancy. These levels are reduced in the liver of ethanol-treated rats, and resveratrol compensates these effects.
\end{abstract}

Keywords: GRP78, BECLIN1, Ethanol, Resveratrol, Gestation, Oxidative Stress

\section{Background}

Pregnancy brings about adaptive changes in the metabolism (1) and physiology of the female body (2). These gestational changes may be associated with disorders like pregnancy-associated liver disease observed in $3 \%$ of pregnant women worldwide $(3,4)$. The higher metabolic demand of the growing fetus and the increased metabolic activity in placental mitochondria leads to increased production of reactive oxygen species (ROS) that puts the mother in a state of oxidative stress (5).

Previous studies have shown that ethanol (Eth) consumption during pregnancy can increase ROS levels and cause liver damage $(6,7)$. Eth induces both oxidative and ER stress in the liver (8). The brain is another organ that is most susceptible to Eth toxicity following its oxidation (9). Chronic administration of Eth is reported to accompany oxidative stress in the adult mouse brain (10).

Unfolded protein response (UPR), as a protective strategy protecting cells against ER stress (11), works toward the reduction of unfolded protein accumulation (12). UPR activity involves the three signaling proteins, activating transcription factor 6 (ATF6), protein kinase R (PKR)-like endoplasmic reticulum kinase, and inositol-requiring enzyme 1 (IRE1) (13). The activation of these proteins occurs upon detachment from an endoplasmic reticulum chaperon, GRP78/BiP (binding immunoglobulin protein), when the accumulated unfolded proteins compete with the receptors attachment to GRP78 $(14,15)$. Neuronal death af- 
ter prolonged activation of UPR following ER stress has been introduced as the mechanism of ethanol neurotoxicity (16).

Autophagy plays a protective role against oxidative stress (17). Beclin1/ATG6 (autophagy-related gene 6) is a key protein in the initiation of autophagy that directly interacts with GRP78 leading to the formation of the BECLIN1Vps34-Vps15 complex and eventually triggering the autophagy cascade (18). Autophagy is shown to be activated by Eth exposure (19).

As a polyphenolic compound, resveratrol (RSV) is found in various foods and drinks such as grapes, plums, berries, peanuts, grape juice, and red wine (20). Numerous beneficial health effects of RSV, such as preventing cardiovascular diseases, diabetes, aging, neurodegenerative complications, and cancer, have been discovered, where RSV acts as a potent antioxidant (21). RSV has shown alleviating effects on fatty liver disease by introducing autophagy in cellular and animal models of hepatic steatosis $(22,23)$. Besides, long-term consumption of RSV is associated with protective effects on optic nerves via activation of UPR pathway effector proteins, CHOP (CCAATenhancerbinding protein homologous protein), and XBP-1 (X-box binding protein 1) (24).

We have previously studied the brain effects of alcohol consumption during pregnancy and have shown that Eth decreased the expression of brain-derived neurotrophic factor (BDNF) in the hippocampus of female rats, which was reversed by RSV (25).

\section{Objectives}

Here, we have hypothesized that pregnancy affects the basal mRNA expressions of genes for autophagy and UPR and affects their response to Eth and RSV.

\section{Methods}

\subsection{Rats}

Twenty-four female Sprague-Dawley rats were purchased from Shiraz University of Medical Sciences' Animal Laboratory with an average weight of $220 \pm 20 \mathrm{~g}$. Subjects were kept in a controlled condition using a 12-hour light and dark cycle. Six nulligravida female rats were randomly selected as the non-pregnant control group. Other animals mated and were examined for pregnancy. The detection of the first vaginal plaque was an indication of pregnancy. Pregnant rats were divided into three groups and treated with normal saline (pregnant control group) and either
Eth or combined Eth and RSV as test groups (Table 1). The doses are selected based on our results from previous studies $(25,26)$. Rats were gavaged by $200 \mu \mathrm{L}$ of the daily treatments for 20 days. At the end of the treatment period, rats were euthanized in a $\mathrm{CO}_{2}$ chamber, and their brains and livers were surgically removed, snap-frozen in liquid nitrogen, and stored at $-80^{\circ} \mathrm{C}$ till the time of RNA extraction. Two brain samples of each group were pooled. All experiments were done in agreement with the animal ethics guideline of Shiraz University of Medical Sciences and were approved by the Vice-Chancellor for Research Affairs with grant number 91-6140.

3.2. Quantitative-reverse Transcription Polymerase Chain Reaction ( $q R T-P C R$ )

RNA was extracted from 50-100 mg of tissues using Trizol reagent (Invitrogen, CA, USA), following the manufacturer's protocol. First-strand cDNA was synthesized from RNA with reverse transcriptase Kit (Fermentas, Maryland, USA). Quantitative analysis was carried out by Real-Time RT PCR using the Power SYBR Green PCR Master Mix (Takara) and Real-Time PCR System (Applied Biosystems 7500, Foster City, CA, USA). qRT-PCR was performed with 40 cycles for amplification. We evaluated single-peak melting curves as specific qRT-PCR reactions. Electrophoresis of qRT-PCR products confirmed the accuracy of the molecular weight of amplicons.

The primers of BECLIN1, GRP78, and ACTIN were designed by AlleleID software (Table 2 ). The $2^{-\Delta \Delta \mathrm{Ct}}$ method was used to evaluate the expression of genes.

\subsection{Statistical Analysis}

Nonparametric analysis of variance (Kruskal-Wallis test) followed by Dunn's post hoc analysis for multiple comparisons was used to determine any significant changes between groups. The significance level was set at $\mathrm{P}$ $<0.05$ for all tests. All analyses were conducted with Graph Pad Prism statistical software (version 7; San Diego, CA).

\section{Results}

4.1. Pregnant Rats Express Higher Levels of Autophagy and UPR Markers in Their Liver

As shown in Figure 1, BECLIN1 encoding mRNA levels in the liver of pregnant rats treated with normal saline were significantly higher compared to the non-pregnant control group (Figure 1A, P-value < 0.001). Similar results were observed for the expression level of GRP78 (Figure 1B, P-value $<0.001)$. 


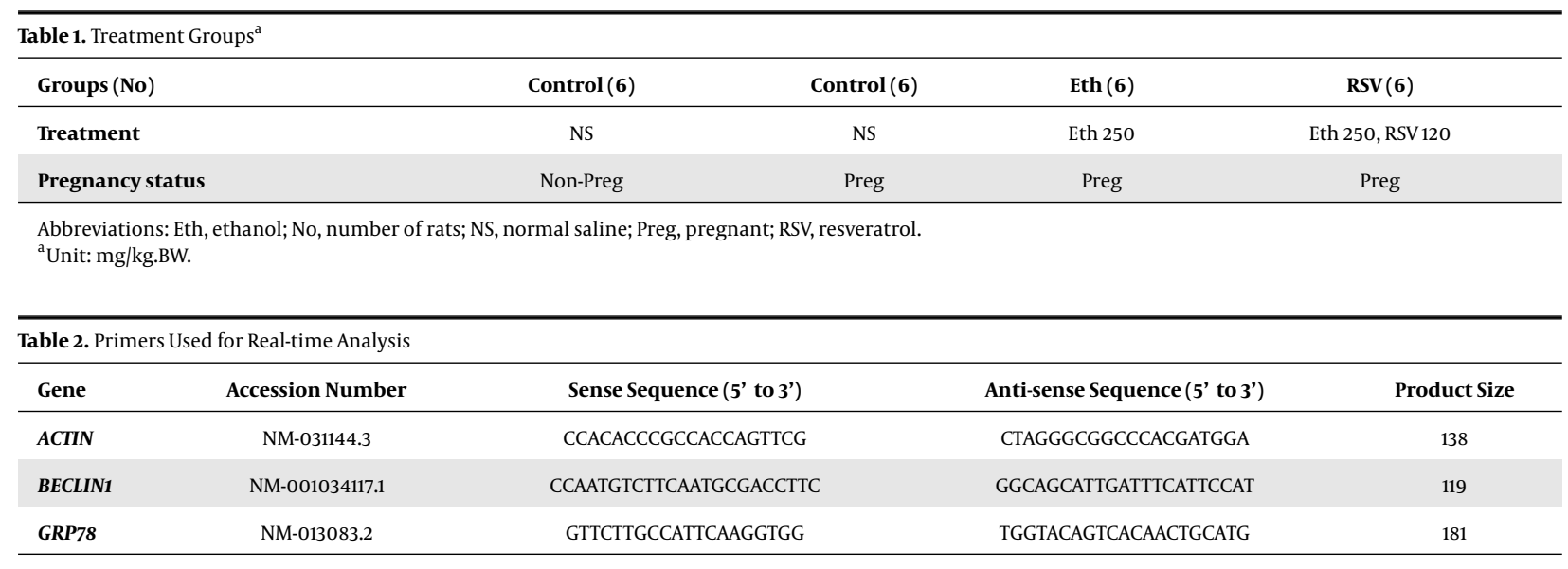

A

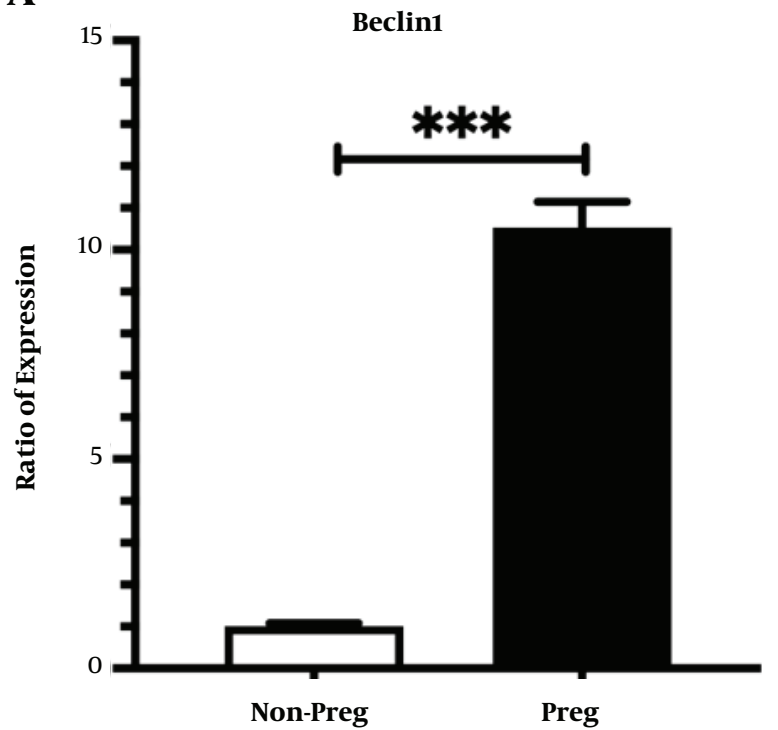

B

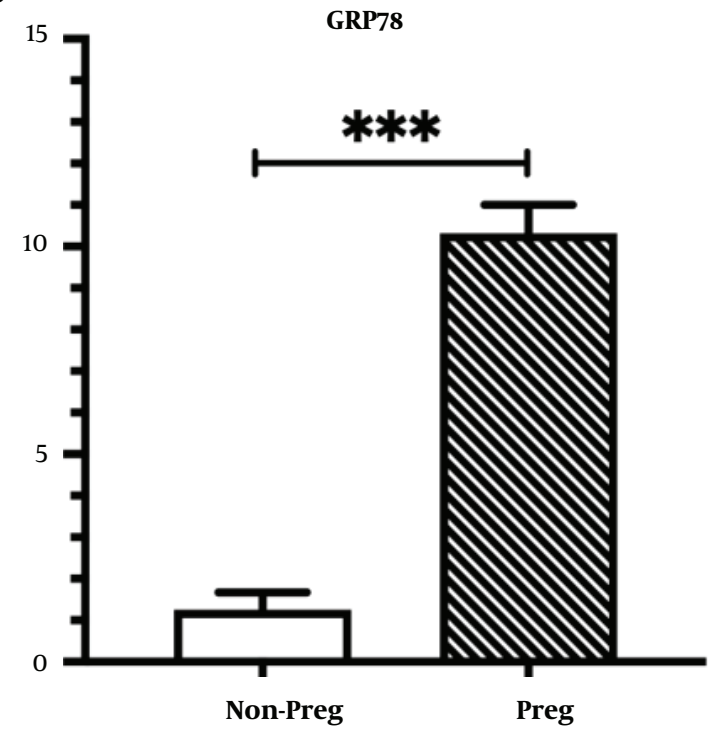

Figure 1. The levels of BECLIN1 and GRP78 transcripts in the liver of pregnant rats compared to the non-pregnant group. Data are presented as the fold of increase in the expression of genes relative to that of the control non-pregnant group and are the mean and SEM of three samples. ${ }^{* * *}, \mathrm{P}<0.001$

\subsection{RSVRestored the Expression of Autophagy and UPRMarkers} in the Liver of Pregnant Rats Exposed to Ethanol

Results of real-time RT-PCR showed that Eth 250 $\mathrm{mg} / \mathrm{kg}$.BW significantly decreased the expression levels of $B E C L I N 1$ mRNA in the liver of pregnant rats compared to the normal saline group (Figure $2 \mathrm{~A}$, P-value $<0.05$ ). However, RSV $120 \mathrm{mg} / \mathrm{kg}$.BW reversed the ethanol-induced decrease in the mRNA levels of BECLIN1. Likewise, the expression levels of GRP78 mRNA significantly decreased in response to Eth $250 \mathrm{mg} / \mathrm{kg}$.BW in the liver of pregnant rats compared to pregnant control rats (Figure $2 \mathrm{~B}$, P-value $<0.05$ ). Addition of RSV $120 \mathrm{mg} / \mathrm{kg}$.BW to Eth could effectively increase the expression of GRP78 to the level of normal saline receiv- ing pregnant rats.

4.3. RSVIncreased the Expression Level of the UPR Marker in the Brain of Pregnant Rats with no Significant Effect on the Expression of the Autophagy Marker

Results of Real-time RT-PCR showed that Eth 250 $\mathrm{mg} / \mathrm{kg}$.BW alone or combined with RSV $120 \mathrm{mg} / \mathrm{kg}$ had no significant effects on the mRNA expression of the $B E$ CLIN1 in the brain of pregnant rats compared to the salinetreated pregnant rats. (Figure 3A). However, in response to oral Eth $250 \mathrm{mg} / \mathrm{kg} . \mathrm{BW}$, the expression levels of the GRP78 mRNA, significantly decreased in the brain of pregnant 

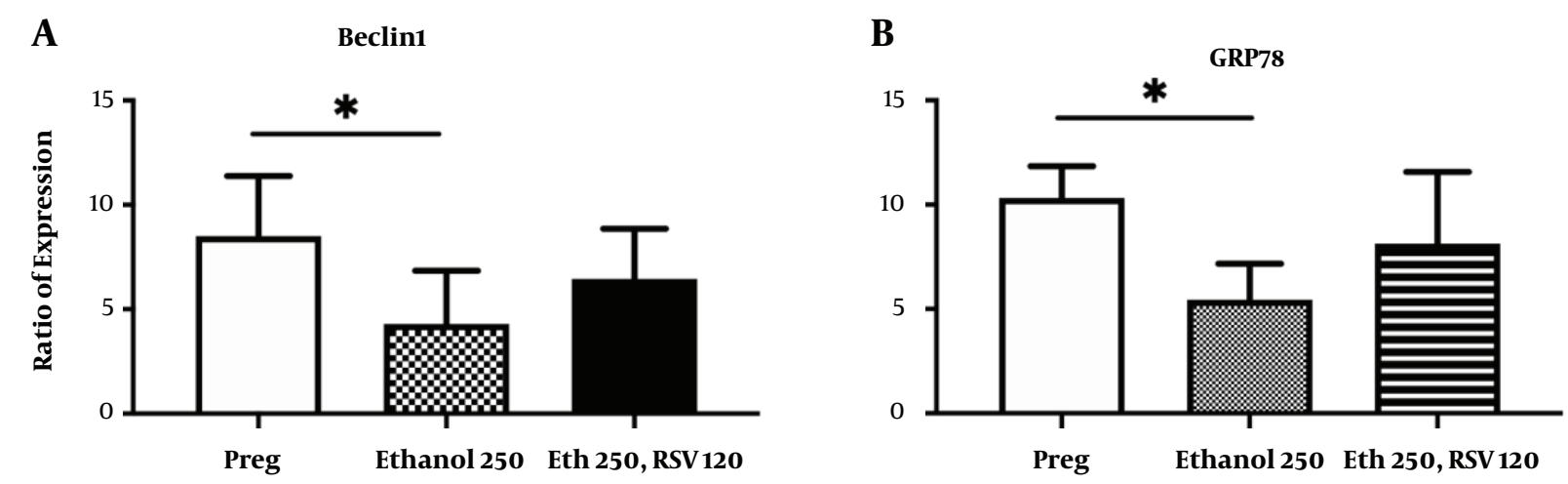

Figure 2. Levels of BECLIN1 and GRP78 transcripts in the liver of pregnant rats treated with Eth alone or combined with RSV. Control pregnant rats were treated with normal saline. Data are presented as the fold of increase in the mRNA level in the treatment groups to that in the pregnant control group. Data are mean and SEM ( $\mathrm{n}=3$ ). ${ }^{*}$, significance at $\mathrm{P}<0.05$ (Dunn's test).

rats compared to saline-treated pregnant rats (Figure 3B, Pvalue $<0.0001)$. Addition of RSV120 mg/kg.BW to Eth leads to an increase in the mRNA expression of GRP78 compare to saline receiving pregnant rats (Figure 3B, P-value $<0.05$ ).

\section{Discussion}

The present study revealed increased levels of mRNAs encoding GRP78 and/or Beclin-1 in the liver of pregnant rats.

GRP78 is a negative regulator of UPR and a strong inhibitor of apoptosis (27). GRP78 has an ER stress response element in the promoter of its gene. Thus, GRP78 is shown to be a marker and regulator of ER stress (28). It is established that pregnancy is associated with ER stress in the placenta and reproductive systems due to induced oxidative stress (29). However, this pregnancy-related oxidative situation on other organs like the liver and brain is not apparent yet. Our results revealed an up-regulation of GRP78 expression in pregnant rats' liver, possibly as a protective function of the liver in stress conditions.

In this study, we have also shown that the expression levels of BECLIN1 were increased in the liver during pregnancy. Pregnancy is associated with increased levels of ROS, and autophagy is triggered by oxidative stress (30). Therefore, the overexpression of the BECLIN1 gene as an autophagy marker in the liver of pregnant rats can be interpreted as a defense mechanism against ROS's gestational overload, which prevents liver injury and reduces oxidative stress through autophagic activity. Autophagy has also been shown in humans' first trimester placental villi, a finding that suggests a pro-survival role for autophagy during pregnancy (31).
Previous studies have suggested that acute Eth exposure induces autophagy in the liver through its reactive metabolites (32). We have demonstrated a decrease in the expression levels of BECLIN1 upon chronic exposure of pregnant rats to Eth. In line with our results, Chao et al. reported that impaired transcription factor EB (TFEB) mediated autophagy promoted the liver injury caused by chronic ethanol intake in mice. Autophagy is also shown to protect hepatocytes from adverse effects of acute alcohol exposure by removing lipid droplets and damaged mitochondria (32). Therefore, lower levels of BECLIN1 we observed in the liver of pregnant rats exposed to chronic Eth are conceivable to decrease autophagic activity and predispose the tissue to injury.

Despite the effect on the liver, Eth failed to decrease the expression of BECLIN1 in the brain samples from our pregnant rats. This finding may be explained by the high basal level of autophagic activity reported in cells with neuronal origin (33).

Recent studies have introduced RSV as an inducer of autophagy. Other investigators have shown that beneficial effects of RSV on the hepatic lipid accumulation induced by ethanol or by high-fat high-sucrose diet associated with the elevated levels of BECLIN1 and the activation of autophagy $(22,23)$. In line with these studies, our results have shown that RSV $120 \mathrm{mg} / \mathrm{kg}$.BW tended to reverse the decreased expression of BECLIN1 in the Eth treated pregnant rats.

In 2018, Li et al. (34) reported that Eth exposure during pregnancy increased the expression of GRP78 and other ER stress markers such as IRE1 $\alpha$ and ATF4 (Activating Transcription Factor 4 ) in the brain of four-day-old offsprings. However, our results demonstrated decreased GRP78 tran- 

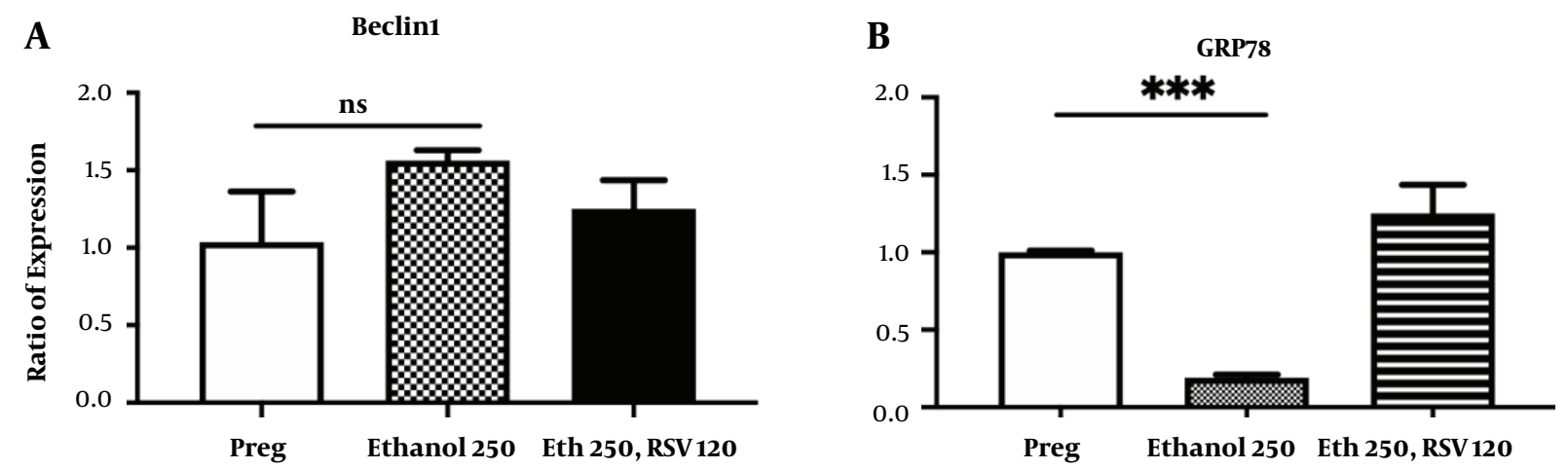

Figure 3. Levels of BECLIN1 and GRP78 transcripts in the brain of pregnant rats. Data are presented as the fold increase in the mRNA level in the treatment groups compared to the control group. Data are presented as the mean and standard error of the mean (SEM). ${ }^{*}, \mathrm{P}<0.05,{ }^{* * *}, \mathrm{P}<0.001$.

scripts in the brain of pregnant rats chronically exposed to ethanol. RSV has shown ameliorating effects on the ER stress induced by free fatty acid in HepG2 cells (35). Further, Gaballah et al. (36), in a rat model of rotenone-induced Parkinson's disease, showed that RSV alleviated ER stress by decreasing the expression of CHOP and GRP78. Despite these findings, our results revealed that RSV recovered the reduced expression of GRP78 partially in the liver and fully in the brain of Eth-treated pregnant rats. The discrepancy between our results and those of other researchers may be attributed to the physiological and metabolic situations in pregnancy, as we have observed a higher basal level of GRP78 expression in the liver of pregnant rats compared to the non-pregnant group. Alternatively, the discrepancy may be due to the effect of RSV on decreasing the oxidative injury via the activation of TFEB and subsequent induction of autophagy as has been shown in endothelial cells (37).

This study is limited by evaluating the effect of pregnancy on autophagy and UPR marker to the transcriptome level. These observations need to be confirmed by evaluating the protein level of these markers.

\subsection{Conclusions}

Here, for the first time, we have disclosed that the basal mRNA levels of autophagy and UPR marker are higher in the liver of pregnant rats compared to non-pregnant ones. Further, we have shown that chronic consumption of Eth disturbs the pregnant levels of these markers and that RSV could recover them.

\section{Footnotes}

Authors' Contribution: SA, GG, EG, YSI, and SS performed the experiments. GG, SS, AE, and AAO wrote the manuscript. GG, SS, and TD performed the statistical analysis. SS and AAO designed the experimental plan.

Conflict of Interests: The authors declare no conflict of interest.

Ethical Approval: All experiments were done in agreement with animal ethics guideline of Shiraz University of Medical Sciences and were approved by the ViceChancellor for Research Affairs.

Funding/Support: This work was supported by grant number 91-6140 from the Vice-Chancellor for Research Affairs of Shiraz University of Medical Sciences, Shiraz, Iran. Shahla Shojaei was supported by grant number 194293 from the Vice-Chancellor for Research Affair of Isfahan University of Medical Sciences, Isfahan, Iran.

\section{References}

1. Cendrowska-Pinkosz M, Dworzanski W, Krauze M, Burdan F. [Changes of carbohydrate and lipid metabolism in women during pregnancy taking caffeine]. Pol Merkur Lekarski. 2017;42(247):38-41. Polish. [PubMed: 28134231].

2. Kovacs CS. Maternal Mineral and Bone Metabolism During Pregnancy, Lactation, and Post-Weaning Recovery. Physiol Rev. 2016;96(2):449547. doi: 10.1152/physrev.00027.2015. [PubMed: 26887676].

3. Kelly C, Pericleous M. Pregnancy-associated liver disease: a curriculum-based review. Frontline Gastroenterol. 2018;9(3):170-4. doi: 10.1136/flgastro-2017-100924. [PubMed: 30046419]. [PubMed Central: PMC6056085].

4. Mikolasevic I, Filipec-Kanizaj T, Jakopcic I, Majurec I, Brncic-Fischer A, Sobocan N, et al. Liver Disease During Pregnancy: A Challenging Clinical Issue. Med Sci Monit. 2018;24:4080-90. doi: 10.12659/MSM.907723. [PubMed: 29905165]. [PubMed Central: PMC6034557]. 
5. Sultana Z, Maiti K, Aitken J, Morris J, Dedman L, Smith R. Oxidative stress, placental ageing-related pathologies and adverse pregnancy outcomes. Am J Reprod Immunol. 2017;77(5). doi: 10.1111/aji.12653. [PubMed: 28240397].

6. Perez MJ, Velasco E, Monte MJ, Gonzalez-Buitrago JM, Marin JJ. Maternal ethanol consumption during pregnancy enhances bile acidinduced oxidative stress and apoptosis in fetal rat liver. Toxicology. 2006;225(2-3):183-94. doi: 10.1016/j.tox.2006.05.015. [PubMed: 16824660].

7. Driscoll CD, Streissguth AP, Riley EP. Prenatal alcohol exposure: comparability of effects in humans and animal models. Neurotoxicol Teratol. 1990;12(3):231-7. doi: 10.1016/0892-0362(90)90094-s. [PubMed: 2196422].

8. Galligan JJ, Smathers RL, Shearn CT, Fritz KS, Backos DS, Jiang H, et al. Oxidative Stress and the ER Stress Response in a Murine Model for Early-Stage Alcoholic Liver Disease. J Toxicol. 2012;2012:207594. doi: 10.1155/2012/207594. [PubMed: 22829816]. [PubMed Central: PMC3399426].

9. Lasek AW. Effects of Ethanol on Brain Extracellular Matrix: Implications for Alcohol Use Disorder. Alcohol Clin Exp Res. 2016;40(10):203042. doi: 10.1111/acer.13200. [PubMed: 27581478]. [PubMed Central: PMC5048555].

10. Gong YS, Guo J, Hu K, Gao YQ, Hou FL, Song FL, et al. Chronic Ethanol Consumption and Thiamine Deficiency Modulate betaAmyloid Peptide Level and Oxidative Stress in the Brain. Alcohol Alcohol. 2017;52(2):159-64. doi: 10.1093/alcalc/agw095. [PubMed: 28182200].

11. Mehrbod P, Ande SR, Alizadeh J, Rahimizadeh S, Shariati A, Malek $\mathrm{H}$, et al. The roles of apoptosis, autophagy and unfolded protein response in arbovirus, influenza virus, and HIV infections. Virulence. 2019;10(1):376-413. doi: 10.1080/21505594.2019.1605803. [PubMed: 30966844]. [PubMed Central: PMC6527025].

12. Senft D, Ronai ZA. UPR, autophagy, and mitochondria crosstalk underlies the ER stress response. Trends Biochem Sci. 2015;40(3):141-8. doi: 10.1016/j.tibs.2015.01.002. [PubMed: 25656104]. [PubMed Central: PMC4340752].

13. Yeganeh B, Rezaei Moghadam A, Alizadeh J, Wiechec E, Alavian SM, Hashemi M, et al. Hepatitis B and C virus-induced hepatitis: Apoptosis, autophagy, and unfolded protein response. World J Gastroenterol. 2015;21(47):13225-39. doi: 10.3748/wjg.v21.i47.13225. [PubMed: 26715805]. [PubMed Central: PMC4679754].

14. Ghavami S, Yeganeh B, Stelmack GL, Kashani HH, Sharma P, Cunnington R, et al. Apoptosis, autophagy and ER stress in mevalonate cascade inhibition-induced cell death of human atrial fibroblasts. Cell Death Dis. 2012;3. e330. doi: 10.1038/cddis.2012.61. [PubMed: 22717585]. [PubMed Central: PMC3388233].

15. Ghavami S, Sharma P, Yeganeh B, Ojo OO, Jha A, Mutawe MM, et al. Airway mesenchymal cell death by mevalonate cascade inhibition: integration of autophagy, unfolded protein response and apoptosis focusing on Bcl2 family proteins. Biochim Biophys Acta. 2014;1843(7):1259-71. doi: 10.1016/j.bbamcr.2014.03.006. [PubMed: 24637330].

16. Yang F, Luo J. Endoplasmic Reticulum Stress and Ethanol Neurotoxicity. Biomolecules. 2015;5(4):2538-53. doi: 10.3390/biom5042538. [PubMed: 26473940]. [PubMed Central: PMC4693246].

17. Bao Y, Pu Y, Yu X, Gregory BD, Srivastava R, Howell SH, et al. IRE1B degrades RNAs encoding proteins that interfere with the induction of autophagy by ER stress in Arabidopsis thaliana. Autophagy. 2018;14(9):1562-73. doi: 10.1080/15548627.2018.1462426. [PubMed: 29940799]. [PubMed Central: PMC6135571].

18. Alizadeh J, Shojaei S, Sepanjnia A, Hashemi M, Eftekharpour E, Ghavami S. Simultaneous Detection of Autophagy and Epithelial to Mesenchymal Transition in the Non-small Cell Lung Cancer
Cells. Methods Mol Biol. 2019;1854:87-103. doi: 10.1007/7651_2017_84. [PubMed: 29101677].

19. Chen G, Ke Z, Xu M, Liao M, Wang X, Qi Y, et al. Autophagy is a protective response to ethanol neurotoxicity. Autophagy. 2012;8(11):157789. doi: 10.4161/auto.21376. [PubMed: 22874567]. [PubMed Central: PMC3494588].

20. Murakami A. Modulation of protein quality control systems by food phytochemicals. J Clin Biochem Nutr. 2013;52(3):215-27. doi: 10.3164/jcbn.12-126. [PubMed: 23704811]. [PubMed Central: PMC3652296].

21. Catalgol B, Batirel S, Taga Y, Ozer NK. Resveratrol: French paradox revisited. Front Pharmacol. 2012;3:141. doi: 10.3389/fphar.2012.00141. [PubMed: 22822401]. [PubMed Central: PMC3398412].

22. Tang L, Yang F, Fang Z, Hu C. Resveratrol Ameliorates Alcoholic Fatty Liver by Inducing Autophagy. Am J Chin Med. 2016;44(6):1207-20. doi: 10.1142/S0192415X16500671. [PubMed: 27627919].

23. Milton-Laskibar I, Aguirre L, Etxeberria U, Milagro FI, Martinez JA, Portillo MP. Involvement of autophagy in the beneficial effects of resveratrol in hepatic steatosis treatment. A comparison with energy restriction. Food Funct. 2018;9(8):4207-15. doi:10.1039/c8fo00930a. [PubMed: 29993072].

24. Lindsey JD, Duong-Polk KX, Hammond D, Leung CK, Weinreb RN. Protection of injured retinal ganglion cell dendrites and unfolded protein response resolution after long-term dietary resveratrol. Neurobiol Aging. 2015;36(5):1969-81. doi: 10.1016/j.neurobiolaging.2014.12.021. [PubMed: 25772060].

25. Shojaei S, Ghavami S, Panjehshahin MR, Owji AA. Effects of Ethanol on the Expression Level of Various BDNF mRNA Isoforms and Their Encoded Protein in the Hippocampus of Adult and Embryonic Rats. Int J Mol Sci. 2015;16(12):30422-37. doi: 10.3390/ijms161226242. [PubMed: 26703578]. [PubMed Central: PMC4691182].

26. Shojaei S, Panjehshahin MR, Shafiee SM, Khoshdel Z, Borji M, Ghasempour G, et al. Differential Effects of Resveratrol on the Expression of Brain-Derived Neurotrophic Factor Transcripts and Protein in the Hippocampus of Rat Brain. Iran J Med Sci. 2017;42(1):32-9. [PubMed: 28293048]. [PubMed Central: PMC5337763].

27. Pyrko P, Schonthal AH, Hofman FM, Chen TC, Lee AS. The unfolded protein response regulator GRP78/BiP as a novel target for increasing chemosensitivity in malignant gliomas. Cancer Res 2007;67(20):9809-16. doi: 10.1158/0008-5472.CAN-07-0625. [PubMed: 17942911].

28. Lee AS. The ER chaperone and signaling regulator GRP78/BiP as a monitor of endoplasmic reticulum stress. Methods. 2005;35(4):373-81. doi: 10.1016/j.ymeth.2004.10.010. [PubMed:15804610].

29. Gao HJ, Zhu YM, He WH, Liu AX, Dong MY, Jin M, et al. Endoplasmic reticulum stress induced by oxidative stress in decidual cells: a possible mechanism of early pregnancy loss. Mol Biol Rep. 2012;39(9):917986. doi: 10.1007/s11033-012-1790-x. [PubMed: 22733488].

30. Chen Y, Gibson SB. Is mitochondrial generation of reactive oxygen species a trigger for autophagy? Autophagy. 2008;4(2):246-8. doi: 10.4161/auto.5432. [PubMed: 18094624].

31. Chifenti B, Locci MT, Lazzeri G, Guagnozzi M, Dinucci D, Chiellini F, et al. Autophagy-related protein LC3 and Beclin-1 in the first trimester of pregnancy. Clin Exp Reprod Med. 2013;40(1):33-7. doi: 10.5653/cerm.2013.40.1.33. [PubMed: 23614114]. [PubMed Central: PMC3630291].

32. Ding WX, Li M, Chen X, Ni HM, Lin CW, Gao W, et al. Autophagy reduces acute ethanol-induced hepatotoxicity and steatosis in mice. Gastroenterology. 2010;139(5):1740-52. doi: 10.1053/j.gastro.2010.07.041. [PubMed: 20659474]. [PubMed Central: PMC4129642].

33. Klionsky DJ, Abdelmohsen K, Abe A, Abedin MJ, Abeliovich H, Acevedo Arozena A, et al. Guidelines for the use and interpretation of assays for 
monitoring autophagy (3rd edition). Autophagy. 2016;12(1):1-222. doi: 10.1080/15548627.2015.1100356. [PubMed: 26799652]. [PubMed Central: PMC4835977].

34. Li H, Wen W, Xu H, Wu H, Xu M, Frank JA, et al. 4-Phenylbutyric Acid Protects Against Ethanol-Induced Damage in the Developing Mouse Brain. Alcohol Clin Exp Res. 2019;43(1):69-78. doi: 10.1111/acer.13918. [PubMed: 30403409]. [PubMed Central: PMC6318008].

35. Li F, Yang Y, Yang L, Wang K, Zhang X, Zong Y, et al. Resveratrol alleviates FFA and $\mathrm{CCl} 4$ induced apoptosis in HepG2 cells via restoring endoplasmic reticulum stress. Oncotarget. 2017;8(27):43799-809. doi: 10.18632/oncotarget.16460. [PubMed: 28415630]. [PubMed Cen- tral: PMC5546441].

36. Gaballah HH, Zakaria SS, Elbatsh MM, Tahoon NM. Modulatory effects of resveratrol on endoplasmic reticulum stress-associated apoptosis and oxido-inflammatory markers in a rat model of rotenoneinduced Parkinson's disease. Chem Biol Interact. 2016;251:10-6. doi: 10.1016/j.cbi.2016.03.023. [PubMed: 27016191].

37. Zhou X, Yang J, Zhou M, Zhang Y, Liu Y, Hou P, et al. Resveratrol attenuates endothelial oxidative injury by inducing autophagy via the activation of transcription factor EB. Nutr Metab (Lond). 2019;16:42. doi: 10.1186/s12986-019-0371-6. [PubMed: 31303889]. [PubMed Central: PMC6604179]. 